\title{
Scaling up Functional Programming Education: Under the Hood of the OCaml MOOC
}

\author{
BENJAMIN CANOU, OCamlPro SAS, France \\ ROBERTO DI COSMO, INRIA and Université Paris Diderot, France \\ GRÉGOIRE HENRY, OCamlPro SAS, France
}

\begin{abstract}
This article describes the key innovations used in the massive open online course "Introduction to Functional Programming using OCaml" that has run since the fall semester of 2015. A fully in-browser development environment with an integrated grader provides an exceptional level of feedback to the learners. A functional library of grading combinators greatly simplifies the notoriously complex task of writing test suites for the exercises, and provides static type-safety guarantees on the tested user code. Even the error-prone manual process of importing the course content in the learning platform has been replaced by a functional program that describes the course and statically checks its contents. A detailed statistical analysis of the data collected during and after the course assesses the effectiveness of these innovations.
\end{abstract}

\section{CCS Concepts: • Applied computing $\rightarrow$ Interactive learning environments; E-learning;}

Additional Key Words and Phrases: MOOC, functional programming, programming education, automated grading, safe program introspection, browser based education platform

ACM Reference Format:

Benjamin Canou, Roberto Di Cosmo, and Grégoire Henry. 2017. Scaling up Functional Programming Education: Under the Hood of the OCaml MOOC. Proc. ACM Program. Lang. 1, ICFP, Article 4 (September 2017), 25 pages. https://doi.org/10.1145/3110248

\section{INTRODUCTION}

At the beginning of 2014 started the preliminary work that led to the creation of the first massive open online course (MOOC) to propose an introduction to functional programming using the OCaml programming language, now known as the OCaml MOOC [Di Cosmo et al. 2015], on the FUN platform $^{1}$, an instance of the Open edX open source project ${ }^{2}$.

It has been running once per academic year starting October 2015, exposing already 8000 learners from more than 120 countries to functional programming and the OCaml programming language.

The goals of this MOOC were at the same time humble and ambitious: expose a wide public to the three key notions of languages from the ML family (functional programming, static typing with type inference and algebraic data types), and ensure that the learners who completed the course master all of them, and are able to write medium sized programs using OCaml. In just seven weeks.

\footnotetext{
${ }^{1}$ The France Université Numérique (FUN) MOOC platform, https://www.fun-mooc.fr/.

${ }^{2}$ http://www.open.edx.com
}

This work is licensed under a Creative Commons Attribution-ShareAlike 4.0 International License.

(C) 2017 Copyright held by the owner/author(s).

2475-1421/2017/9-ART4

https://doi.org/10.1145/3110248

Proc. ACM Program. Lang., Vol. 1, No. ICFP, Article 4. Publication date: September 2017. 
Reaching these goals turned out to require a huge effort, especially for building the integrated environment that allows learners to do all the exercises directly in the browser, and for designing and implementing the automatic grading framework that is necessary to evaluate the exercises in a safe way. Even the apparently innocuous task of uploading the course content to the MOOC platform became a challenge, when the graphical user interface that is offered to perform this task turned up to require thousands of gestures just for the initial upload.

The solutions designed to solve these challenges are faithful to the type-safe, static checking philosophy that underlies the ML family of languages: an innovative library of highly parametric test functions and combinators has been designed to allow writing tests and grading code concisely and type safely, and to force the test designer to handle all error cases; a unique integrated development environment has been developed to run fully in the browser, avoiding the hassles and bottlenecks of remote execution servers; even the course description itself has been built as an OCaml program, whose execution produces a ready-to-import archive that is statically guaranteed to have no dangling pointers.

In this article, we describe the choices made, the challenges faced, and the solutions found during the journey that led to the successful first run of the course. There are innovations both on the educational and the technical side.

We will start by exposing the main goals of the OCaml MOOC, and explaining how the course content has been organized in Section 2. Section 3 presents the design of the exercise platform that has been developed for the course, taking into account the external constraints imposed by the particular MOOC platform that was used. The format of the exercises is explained through a first, simple example. Sections 4 and 5 contain the main technical contributions of the paper: they describe the challenges that arose while developing the exercises, and the elegant solutions that could be provided, leveraging all the power of the strongly typed, functional programming model offered by the OCaml language. Of particular relevance is the collection of higher order parametric functions and test combinators that were designed to allow writing automatic graders in a concise, complete and type-safe way. Section 6 gives a quick overview of another experiment that was made when developing this MOOC, bypassing the official MOOC authoring tool from the platform, in order to use a MOOC description DSL instead. This approach saved lots of time and trouble. Section 7 presents a statistical analysis of the results of the first two sessions of the OCaml MOOC, that ran in October 2015 and in September 2016. Finally 9 concludes with the lessons learned and future works.

\section{DESIGNING THE COURSE}

Teaching introductory courses in programming is a significant challenge which has always been at the center of heated debates in Computer Science departments. There are strongly differing opinions on what language one should use [Bryant et al. 2010; Dewar and Astrachan 2009], what features of a programming language should be emphasized [Felleisen et al. 2015], what kind of exercises are the most appropriate for retaining attention and fostering participation, and what development environment is most adapted for newcomers.

Teaching introductory courses in functional programming may seem to simplify issues a bit, but in reality it also raises quite a few questions. Shall we be pure or impure? Shall we use typed or untyped languages? Shall we use a subset of the language or the full language? 
Teaching a massive online course adds to the complexity of the course design space. New issues are raised such as the automated evaluation of learners' code, the wide variety of learners, with different backgrounds and expectations, and the choice of the development and execution environment offered to them.

For the OCaml MOOC, some of these decisions were obvious: the focus is on functional programming, and the language chosen is OCaml. Many others were extensively debated among the community of OCaml teachers that offered advice when the course was in its early stages of design.

With no pretension of possessing the ultimate truth, we describe here the rationale of the final structure of the course that was chosen, and how the design of the course and the exercise environment were nicely intertwined.

The main educational objective of the course was to familiarize a wide public to the three key notions: functional programming, static typing with type inference and algebraic data types. These notions are the common pillars of all the programming languages of the ML family [Milner 1978], and despite superficial differences, they are remarkably stable across all the known dialects.

For this reason, after an introductory Week 0 providing broad motivations and background, the structure of the course, shown in Figure 1, has been designed to fully focus the four core weeks of the course (1 to 4 ) on these notions. Expressions, definitions, functions and recursion are introduced using only basic data types in Week 1, that also exposes the learners to the type inference mechanism; Weeks 2 and 3 gradually introduce user defined types, algebraic data types, the traditional list and trees and the notion of type polymorphism; finally, Week 4 is dedicated to functions as first class citizens, introducing partial application and higher order functions, with the classical map and fold operations on lists and trees. Particular care was taken to avoid possible confusions with notions coming from the usual imperative programming languages: for example, the term variable is never used, and identifier is employed instead. Finally, students get their first notions of functional code architecture in the last course week about the module system of OCaml.

\begin{tabular}{|r|l|}
\hline Week & Content \\
\hline \hline 0 & History and motivation, course overview \\
\hline \hline 1 & Basic types, expressions, functions, recursion \\
2 & Simple type definitions, tuples, records \\
3 & Algebraic data types, lists, trees, polymorphic types \\
4 & Higher order functions, map, fold \\
\hline \hline 5 & Imperative features: I/O, exceptions, mutable data \\
6 & Modules, functors, abstract data types \\
\hline \hline 7 & Project \\
\hline
\end{tabular}

Fig. 1. Summary of the OCaml MOOC content

All these notions are extensively put into play in a dense set of problems and exercises: the learners can work on them in a full fledged development environment that sports a toplevel (or REPL) running in the browser. This approach allows the course to go through the material of these four core weeks without introducing any I/O functions, and without spending an ounce of the learners' energy on installing a local environment on their machines. Pointers to the documentation 
for installing a standalone OCaml development environment are of course provided, but nothing, not even the final project, requires it: special care has been taken to ensure that everything can be done entirely in the in-browser exercise environment.

Up to Week 4, the content covered is the pure, polymorphic, strongly typed functional core of OCaml with algebraic data types. According to some learners' feedback, it provides a very satisfactory introduction even for users of other dialects of the ML family, like F\# and SML.

The last two weeks introduce the notions needed for real world programming: input / output and compilation units for writing standalone programs, exceptions and mutable data for the performance hungry, and modules, functors and abstract data types for programming in the large. To complete the course, a choice between two full-fledged projects is offered to the learners.

Our stated goal was not to rank learners on how quickly they did their homework, but to retain them all along the course and to make sure that those who completed it successfully became able to write medium sized functional programs of reasonable complexity using OCaml.

To this end, building on ideas reported by previous experience reports in the literature [Karavirta et al. 2006; Laakso et al. 2005], we decided to invest a great amount of energy in providing a state-of-the-art exercise environment, and lift the usual submission limit and deadlines on the exercises. Learners were able to try and resubmit their solutions as many times as they wanted, until the end of the course.

\section{THE EXERCICE ENVIRONMENT}

\section{Effective teaching may be the hardest job there is.}

\section{William Glasser}

As seen in the previous section, the OCaml MOOC gradually introduces newcomers to functional programming, exposing the learners step by step to notions that are quite new if the only previous experience in programming has been obtained using imperative languages. Hence, minimizing the distractions coming from the difficulties of setting up and learning to use a proper development environment was our top priority, to maximize the time and energy that the learners spend understanding the relevant new notions, and collaborating among themselves.

This section first details and motivates our design choices, then provides an overview of the environment interface and implementation, and finally presents the process of exercise authoring.

Design Choices. From the start, there were two key points on which we decided to make no concessions. First, we wanted all the learners to have exactly the same development and execution environment in front of them. And we wanted learners to avoid as much as possible all installation hassles to get this environment running. Second, we decided to build a system that provides quick feedback to the learners by automatically grading the exercises, which is now broadly recognized as a significant catalyst to improve learner proficiency [Gramoli et al. 2016; Milojicic 2011].

There are many ways to tackle these topics. One can dedicate a significant amount of time at the beginning of the course to help the learners install a special developer environment on their machines [Miller et al. 2014]. This approach can work well when the programming language taught has already a large user base, and when the very same developer environment is easily available across all platforms. 
Another possibility is to select and install a specific development environment on some remote servers, and provide the learners access to this environment via a web front-end, typically an Ajax console. This approach is used by a variety of online programming platforms [Papancea et al. 2013; Piccioni et al. 2014; Pritchard and Vasiga 2013], exactly because it avoids installation hassles for the learners, and presents all of them with the very same environment. But it comes with a series of significant shortcomings. For instance, the servers must be able to stand the peak load that arises when all learners work together, so any downtime or overload on the servers is a real problem. Moreover, the learners' code needs to be properly sandboxed to ensure that the grading platform is not compromised by malicious code.

Neither of these approaches were satisfactory for a course that was going to use the OCaml programming language on a MOOC platform on which very little control was provided.

So, we decided to go much further, and pursue an original solution: move everything into the browser, with a real client-side only environment allowing development, execution and testing of the learner's code. To the best of our knowledge, this had never been done before.

We started from the existing experiment of the in-browser toplevel TryOCaml ${ }^{3}$, available for OCaml thanks to the js_of_ocaml [Vouillon and Balat 2014] OCaml to JavaScript compiler. On top of it, we built a complete, standalone development environment, with a full-fledged code editor, and a flexible, type-safe grading environment that allows to write easily and safely program tests that will be executed directly in the learner's browser.

While it can be argued that the fact that the grader runs on the learner's machine is a potential security concern, as learners could in principle try and reverse engineer the JavaScript code and our basic cheating protection scheme to circumvent the tests and always get the maximum grade, in practice this turned out to be a non issue. And anyway, it would have been easy to rerun offline the graders for all the learners.

The real challenge we had to face was to find a way to make sure that the test code used to grade the exercises would never crash in the learner's browser, no matter how broken or malicious the learner's code might be. Indeed, we had no way to do a post-mortem analysis of any particular run on the grading platform, because, being in the learner's browser, we had no access to it. Strong typing was our lifesaver.

The result is a unique approach that allowed us to get unprecedented scalability, avoid altogether all the installation, administration and security issues posed by a centralized and safe grading server, and provide immediate feedback to the learners, who reported an extremely high degree of satisfaction in the final survey.

Technical overview. The overall structure of the exercise environment developed specifically for the OCaml MOOC is shown in Figure 2, that reproduces a typical exercise page integrated in the FUN/Open EdX platform.

Besides the usual navigation menu and discussion thread, that are standard and come with the platform, one finds the text of the exercise followed by a text editor, initialized with a template solution specific to the current exercise.

The text editor component is based on the ACE editor ${ }^{4}$ - written in JavaScript-which has been customized for OCaml development. It integrates the de facto standard indenter for OCaml code (ocp-indent). This allows us to provide by default a forced-indentation mode that helps beginners to get used to the syntax and scoping rules.

\footnotetext{
${ }^{3}$ https://try.ocamlpro.com/

${ }^{4}$ https://ace.c9.io/
} 


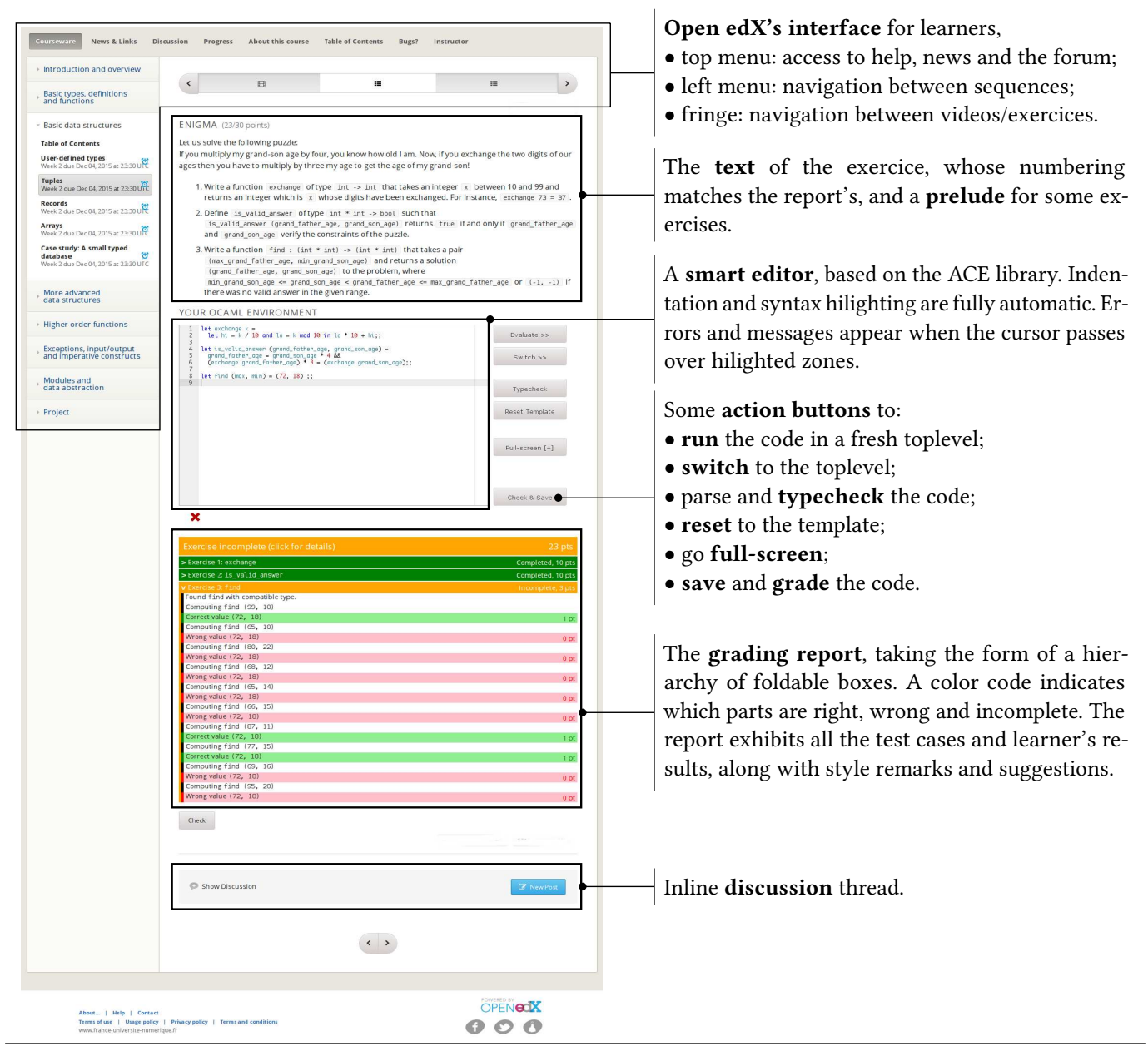

Fig. 2. The exercise platform integrated into Open edX

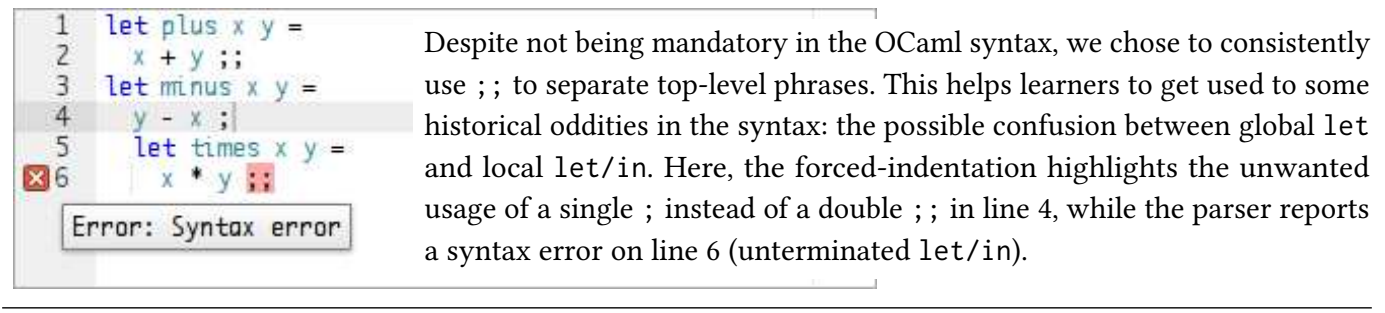

Fig. 3. Forced indentation and error-reporting in the editor

Learners are able to check the syntax and types of their code right away with a single click on a button located on the right of the text editor. After clicking the button, the text editor flashes in green or red, depending on the success or failure of the checks. Syntax and type errors are turned 
into annotations in the text editor, and passing the cursor over the faulty code displays the error message in a small pop-up. The actual presentation is illustrated in Figure 3.

Once validated, the learner's code can be loaded in a toplevel, to allow interactive testing of their solution. This toplevel is an extension of the well-known TryOCaml toplevel, which runs fully in the browser and allows a fluid interaction, without relying on remote servers.

The most notable improvement is the use of the WebWorker API provided by recent browsers in order to execute the toplevel in its own thread. This allows the learner to kill a looping computation without reloading the whole page-which in bad circumstances might imply learners loosing their unsaved work. Using a WebWorker also provides better control of the standard output flow, which might, in extreme cases, overflow the browser with redraw instructions and make it freeze badly.

Once the learner is happy with the code of the solution, it is time to click on the Check button. The code is then automatically graded, locally in the browser, then saved alongside the grade on the MOOC platform. A detailed report of the grading results is displayed below the exercise, in the form of a set of foldable boxes that highlight, one by one, all the aspects of the tests performed for this particular exercise, allowing the learners to quickly identify their mistakes.

Format of the Exercises. While preparing a programming course with automatic grading, one notably time-consuming task is writing the test-suite for each exercise. This is visible in Figure 23, at the end of the article, that sums up the amount of code written for the various components developed for this MOOC. In the design of the grading platform, we took care to streamline this process as much as possible.

From the teacher's point-of-view, adding an exercise consists simply in providing six source files, described in the following, some of them being optional. A sample exercise is given in Figure 4.

The first required file is an HTML fragment which describes the task. It might be accompanied by two optional OCaml files: template.ml and prelude.ml. The former will be used to initialize the text editor and the latter will be visible to the learner and pre-loaded in the environment where the learner's code will be evaluated. Typically, we used the prelude file during the MOOC to provide some custom data types, or example data for learners to test their solutions.

Then, the author must provide two OCaml files, one being the test-suite itself (test.ml) and the other a solution to the task (solution. $\mathrm{ml}$ ). The solution is mandatory: it is used offline, before deploying the MOOC, in order to validate the grader and compute the maximum score. To simplify and automate this task, we developed an OCaml script that simulates the grading environment outside of the browser. The solution is also used online by the test-suite to compare its reference results with the ones of the learners on randomly generated inputs.

A last optional OCaml file is prepare.ml. It is a complement to prelude.ml whose source code will not be visible to the learners. Typically, we used this file to redefine and instrument some basic function, for instance for counting how many times the function Pervasives. compare was called when grading a comparison function. Examples of this usage are detailed in Section 5.

The actual OCaml library that we designed for writing the test-suite is detailed in the next section. But let's first describe the low-level interface. In the online platform, the test.ml file will be evaluated in an environment that contains:

- all the bindings of prelude.ml and prepare.ml ;

- the AST of the learner's code ;

- the evaluated learner's code (isolated in a module);

- a reference results that is detailed below ;

- type safe introspection primitives (cf. next section);

- the high-level grading library (cf. next section). 
pilot its control, access and restrain its typing and execution environments, and manipulate its values directly, including functions. Everything remains safe thanks to static typing, and we will see that it also helps debugging graders.

To make this possible, the library we designed for writing automated graders is composed of three parts: an intermediate language for formatting reports; a set of safe primitives to run the learner's code and introspect the results; and a grading library that helps automating the process of introspecting the learner's code to produce reports, combining the previous two parts.

In this section, we give a technical overview of these building blocks, explaining how they answer the challenges listed in the first paragraph.

An intermediate language for reports. Instead of simple output artifacts to explain their grades to learners, reports are first class elements of our grading system.

The report language:

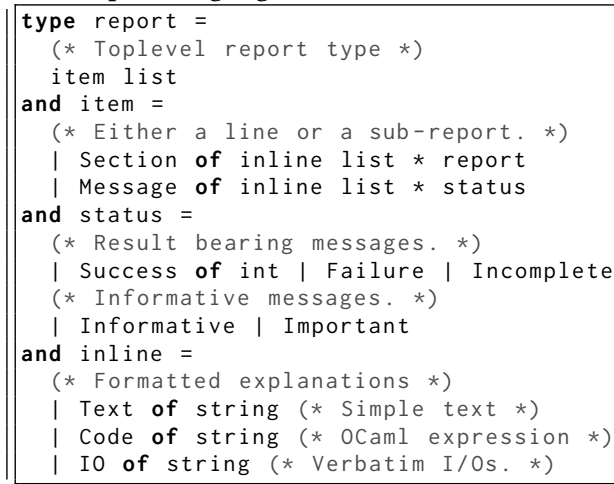

Fig. 5. The intermediate language for reports

They are assembled in a proper intermediate language, whose complete syntax tree is given in Figure 5. Reports can be hierarchically structured, usually matching the corresponding exercise texts. Node elements are either labeled sections or sequences. Leaf elements bear both explanations for the learner and semantic grading information: success or failure, and score. Of course, higher level report building functions are used to build reports in a more concise and homogeneous way.

The benefits of using an intermediate language for reports are manifold. First, it allows us to produce both textual and HTML versions of reports. Then, it allows us to tweak the HTML for inclusion in either Open edX or our standalone platform. But of course, all this could have been achieved by simply using different implementations of an abstract report API. The real argument is that reports can be introspected. This can be used for various tasks, such as skipping an exercise if the previous one was unsuccessful, redact or filter a report a posteriori, compare several reports in a smart way thanks to the structural information, or gather reports in order to compute statistics.

Safe execution and introspection of learners' code. We rely on OCaml's toplevel mechanism, whose main use is the interactive OCaml evaluator ocaml, a tool used by beginners to apprehend the language as well as experienced users to quickly try short fragments of code. But the toplevel system can be used in various other ways. First, it can be linked with any other compiled OCaml unit to build custom evaluators which are aware of a specific set of libraries or application components. In the same vein, it can be plugged in a running application as a debug port. Finally, it can be used to load new code in a running application, using OCaml as its own scripting language. For this, 
the main program can introspect the toplevel environment and obtain its values as if they were its own. But this interface is not typed. All values have type Obj.t, an internal OCaml type castable to any other without any check. The introspection library we describe here is basically a typed layer over this mechanism.

In order to use values from the toplevel properly, we had to define a safe type cast operation for OCaml. This is not generally possible, since OCaml does not keep run time type information. In fact, OCaml drops most of the type information just after typing to keep the bare minimum necessary for its language constructs and memory manager. Experiments have been led to verify that a value is compatible with a type by using only its representation and this minimal information [Henry et al. 2012]. But in our context, we really want to check that the learner's code has the expected types, not just that it will not make our grader crash. So we had to actually unify the types of the learner's code with our expectations.

Obtaining the types of the learner's values is not difficult. The OCaml toplevel works by calling an embedded OCaml compiler to produce a small chunk of byte-code (or assembly depending on the chosen flavour) for each sentence, which is then dynamically loaded and run. We just had to expose the typing environment of the compiler so that our introspection library can obtain the type of any symbol.

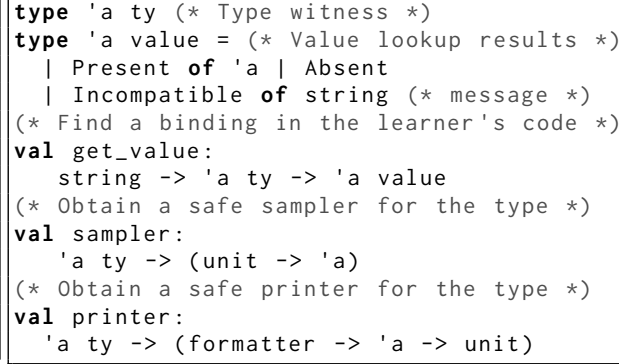

Fig. 6. Introspection interface

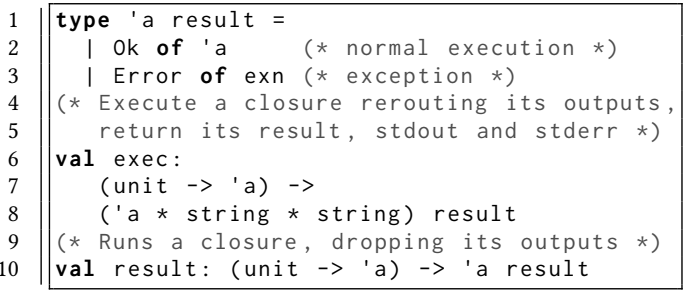

Fig. 7. Code execution primitives

We also had to provide a way to reflect the types expected by the grader at run-time in the same format. For this, we require a little help from the grader author. When she wants to extract a value from the learner's code under some type $\tau$, she has to insert a literal of the form [\%ty: $\tau]$. When compiling the grader, this literal is translated to an expression that has type $\tau$ ty, and that evaluates to a type term for $\tau$. In other words, a value of type $\tau$ ty is a run-time reflection of its argument $\tau$.

Figure 6 gives the interface of our introspection library. It shows the 'a ty type and the primitives to use it. The main one is get_value, which achieves our goal of values transiting between the learner's evaluated code and compiled grader code safely. It takes a type literal and returns Present with a value of the expected type if and only if the value has been found and the reflected type expectation is actually an instance of the actual type of the value in the compiler's environment.

These type witnesses are also used to derive safe printers and samplers, to produce readable reports and generate test cases. Printers simply output values in OCaml syntax. Samplers are built by derivation of the types' structures, and using a table of custom samplers indexed by type constructors. The table initially contains samplers for built-in types, and is alterable from the grader. 
The author just has to define a function sample_t: unit $\rightarrow t$ to update the sampler for values of type $t$ for the rest of the grading. Advanced ways of customizing random sampling are also provided, but this very simple approach is often enough, as in the example of Figure 4.

Once we have this ability to lift the learner's functions in the grader, we have to run them on test cases. Since OCaml is not a purely functional language, this has to be done carefully, in order to handle side effects properly. This is done through the primitives of Figure 7 that call a function, capturing its exceptions and outputs.

Automated grading combinators. Provided the two already presented components, one can already write graders. The top part of Figure 8 gives a fully handwritten grader for the integer successor function. The set_result function computes the score and generates the HTML from the report, and sets the result mentionned in Section 3.

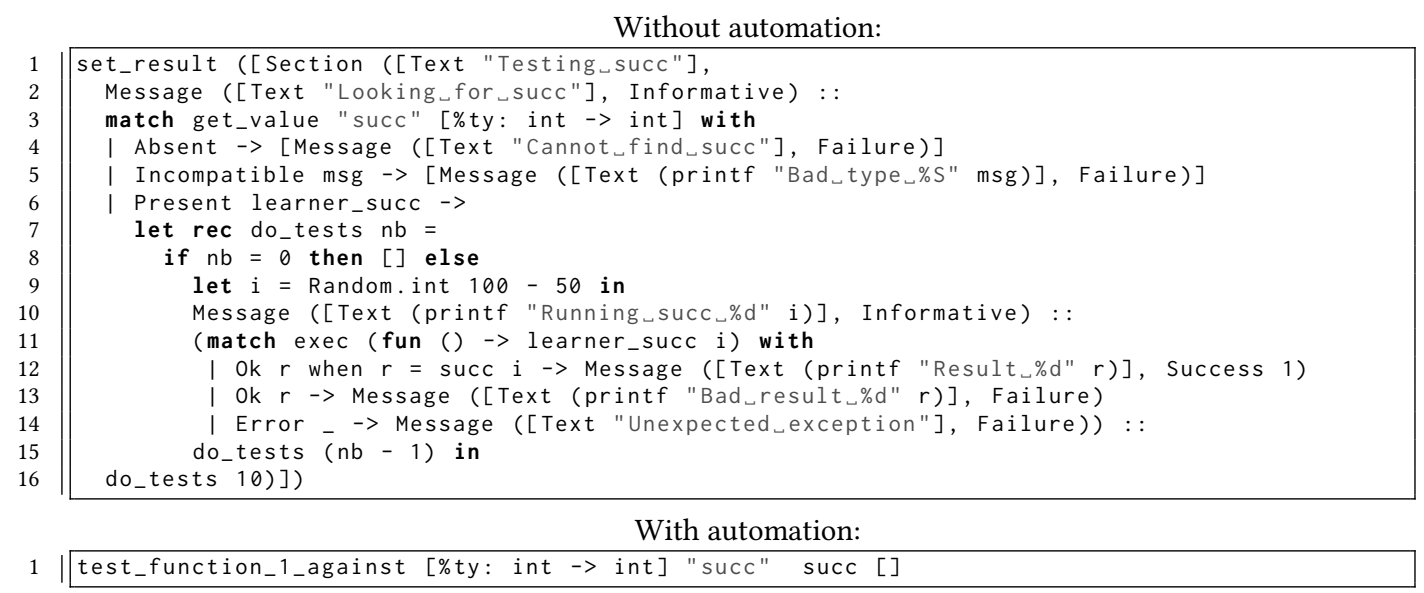

Fig. 8. Grading a function with and without automation

This example is interesting because it shows that the combination of static typing and forced pattern matching prevents lots of errors at compile time. In other automated grading systems, it is not uncommon to have to test the grader in advance on fake submissions to obtain an acceptable level of confidence in the grader, and then to still have to debug graders in production. In practice, during the MOOC, we never had to fix bugs in graders. The only adjustments we had to do were to change the bias of some test case generation or to make some grading policy more flexible or more consistent with the exercise text. And when such a change was needed, we never hesitated to update the graders to enhance the experience of the learners, thanks to the high level of confidence provided by the static checks.

Looking at this example, it seems evident that many parts of it could be automatically generated. That is precisely the goal of our automation library. A first example can be seen in the bottom part of Figure 8, whose one liner code is equivalent to (and actually a bit better than) the handwritten version. In the end, the ratio between a grader and the associated solution is of about 3 , as shown in Figure 23 at the end on the article, which is really reasonnable, according to our past experiments with other automated grading systems. Besides factorizing code, the library also helps homogenizing the format and messages of reports among graders. A complete overview of this library would be out of the scope of this article. We will just present its structure and a few selected functions. 
Syntax checking. In an introductory course such as this MOOC, learning the syntax is an important step. The grading library provides a set of customizable AST traversals that enable checking relevant aspects of the syntax of the student's code. They allow, for instance, to verify that the student has indeed written two nested for loops, when the exercise so requires.

This mechanism is also used to prevent some basic forms of cheating, as shown in Figure 4 with function (ast_sanity_check code_ast). Its job is to prevent the grader from executing if the learner's code contains things that may break the system, such as foreign function declarations or calls to forbidden internal modules.

In the same vein, another use case that occurred several times during the MOOC is restricting the free variables that are allowed to appear in the learner's code. This way, we can ensure that the learner will not, for instance, use predefined combinators of the standard library, when asked to implement the basic operations over lists. One could argue that this last task could also be done by removing the List module from the environment of the toplevel. But the control that the library provides is much finer, since the grader can apply different AST checking policies for different parts of the exercise.

Non functional toplevel definitions. The most common exercise paradigm is to ask the learner to write a function, and then to grade it by calling it with a randomly sampled set of parameters and comparing the outcomes with the expected ones. But sometimes, exercises ask the learner to produce a toplevel value that is not a function.

This is rarely just to ask the learner to produce a constant value. There is most of the time a context-specific reason, and the grader author has to improvise with the tools at hand to implement it. Let us detail two examples that arose during the MOOC.

At the very beginning, learners did not know how to write functions. Yet, we really wanted to make them apprehend the exercise environment as soon as possible, so we decided to write exercises as soon as they were able to write simple let bindings. So in these early exercises, we simply asked them to assign the result of simple computations to global variables. In order to randomize the exercises, we resorted to a very simple trick. As explained in Section 3, the prelude is loaded before both the learner's code and the grader. Thus, we assigned a variable in the prelude to a random value, and asked them to use it in the computation of another binding. For instance, one of the questions was to produce a variable x_power_8, whose name speaks by itself, given an existing $\mathrm{x}$. To help learners debug their solution, the chosen value for $\mathrm{x}$ is then provided at the beginning of the report.

Then, at some point, the learners were taught how to read code written by other people. For exercising this, we gave them the implementation of a small in-memory database, in which we introduced a bug on purpose. The learners were then asked to find the bug, and to produce a sequence of database queries that would lead to the expected corruption, in a global variable. To check the solution, the grader simply fed the sequence to the originally flawed implementation and to the fixed one, expecting a difference.

Complexity. Along with simple exercises, we gave learners a few more complex problems to solve per week. These problems sometimes required them to implement a specific algorithm, respecting its complexity. 
A first trivial idea to check that some algorithm has the appropriate complexity is to run it on a well chosen instance, and to verify that it terminates inside a well selected time window. This is inappropriate in our case for two reasons. First, remember that everything runs inside the learner's browser, so computing a sensible time limit is not that easy. Then, JavaScript does not allow the programmer to interrupt a piece of code, unless it is explicitly written in continuation passing style. Hence, we would have either to kill the grader completely, or to wait for the function to complete, which in case of a wrong complexity could virtually take forever.

As a result, estimating the complexity of a function using its running time was not an option. Instead, the technique we used in the MOOC consists in the manipulation of the environment of student code, so that its execution actually records the number of performed operations. We detail two actual examples from the course.

To give a little more challenge to the previously mentioned x_power_8, we asked the learners to compute it using only three multiplications. So we used the syntax checking mechanism presented in a previous paragraph to make sure that the only free variable of the expression was the operator $(*)$, and we redefined it in the hidden prelude to increment a global counter. Then, the grader simply had to check the value of this reference after the learner's code had been run.

A more complex example arise when learners had to write find: ' a array $\rightarrow>$ ' a $\rightarrow$ int, a function using dichotomy in order to find an element in a sorted array in logarithmic complexity. To check the complexity of this function, we used the same technique as above, and redefined the Array module so that all its operation would increment a global counter.

Then, the grader must decide if the number of operations performed for each test case is correct, and for this, two options are possible. A first possibility is to give a very strict and choice free specification of the algorithm to the learners, and to require them to do the exact minimum of operations. A second possibility is to record the operation counts for all test cases, and to perform a regression analysis to check that the complexity is the expected one and stays within an appropriate constant multiplier range. In practice, it appeared that learners were less confused by the first approach.

In general, the feedback from learners was that grading reports were more useful when the grader is strict than when it is flexible. During the MOOC we actually updated a few exercises to make their graders less accepting, at the demand of learners.

In the end, this approach requires a little more work that an estimation based on the actual running time, but it is strictly better in terms of learner experience, since it actually counts and reports the steps of interest, and it can use effectively even on small inputs, limiting the grading time. As a bonus, it also provides a better alternative to timeouts, by stopping the computation using an exception after some maximum number of operation has been reached.

Side effects. A full week of the MOOC was dedicated to the imperative constructs of OCaml, such as exceptions and mutable structures. Exceptions are transformed in a result type, as already shown in Figure 7, so nothing special has to be done for them. To examine effects on mutable structures, we use the callbacks of function graders shown in Figure 9. Basically, before_reference will save the state of the structure before launching the solution, then before_user will save the reference result and restore the structure so that both the learner's code and the solution's are launched in the same state, and finally, after will save the the learner's result and restore the 


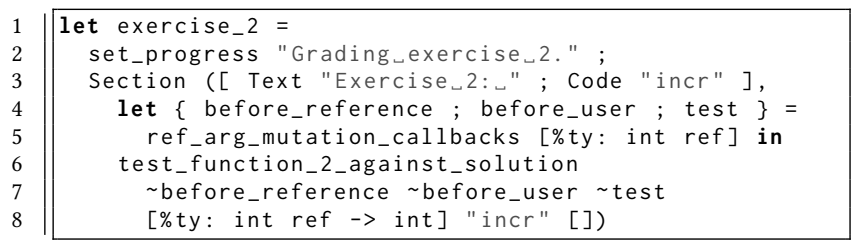

Fig. 10. Grading mutations

structure to its original value. After that, test will simply have to produce a report using exactly the same mechanism as the one for result values.

To avoid reimplementing the technique for each exercise, the library provides a series of callback generators that cover the built-in mutable types of OCaml. For instance, Figure 10 gives an example from the MOOC that uses a callback generator for checking effects on a reference passed as argument. The exercise asks the learner to write a function that increments a reference and returns its previous value. The report will contain one line for the result and one line for the side effect for each test case (if the result type were unit, the results would be ignored).

I/Os. Even if they are also implemented as imperative constructs in OCaml, I/Os are conceptually less difficult to handle than mutations. After all, inputs and outputs can be considered as parameters and results of the the learner's code to grade. That is exactly how it works in the grading library, as already shown in Figure 7. The exec function will trick the learner's code into producing its outputs into buffers, whose contents can simply be tested as if they were result values. Conversely, inputs can be faked by filling input buffers before running the learner's code.

But grading I/Os properly is difficult for another reason. This is the exception to the claim we made a few paragraphs above that learners prefer strict graders and precise specifications. Most of the time, outputs are just a way of presenting the result, and requiring character-perfect output would just increase the risk of frustration among learners. For this, the library provides a group of customization primitives, as shown in Figure 11. They basically help reducing the output to its canonical minimum, for instance by trimming and filtering unwanted characters, before comparing it to the reference output. In practice, the learner will be allowed to mistake a comma for a semicolon, separate words by more than one space, leave a trailing semicolon, etc.

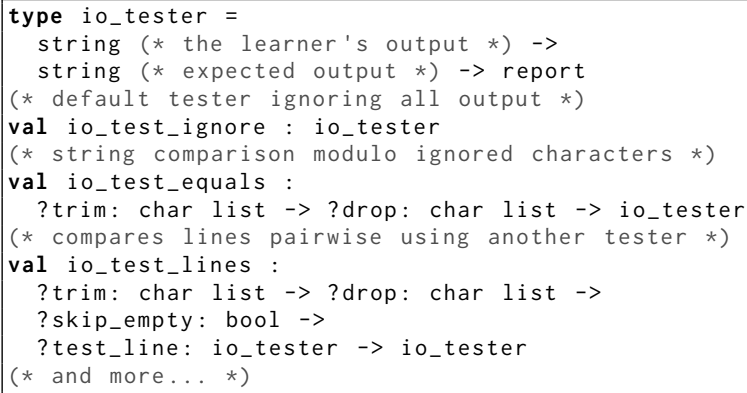

Fig. 11. Output testing primitives

However, there are times when I/Os should be graded strictly. For instance, one of the final projects of the MOOC asked learners to write a parser for text files, recognizing words, punctuation, and paragraph breaks. This kind of exercise requires a little bit more thought from the grader author, at least if she want learners to enjoy the exercise. It is one of the examples that really validated 


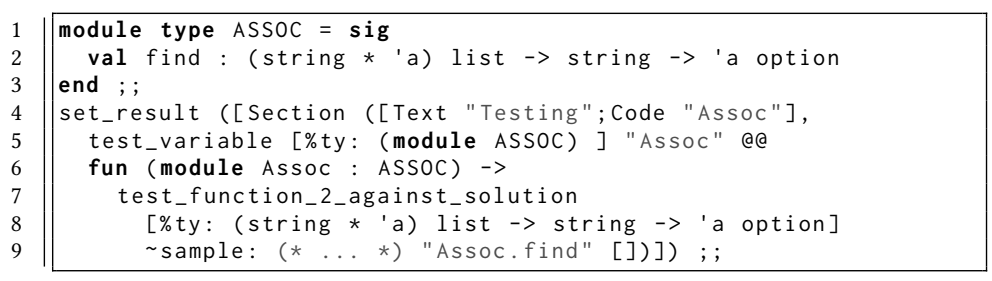

Fig. 12. Simple module exercise

the design of the grading library. By providing an important level of concision, the first version of the grader was very quick to write. After a few tests, we thought that learners would have a hard time finding their bugs, unless we split the parsing process into basic steps returning intermediate parsing states. This way, we could simply compare these states with the ones of our reference parser. We did not hesitate to implement this update, thanks again to the level of concision. This way, learners were able to find their bugs as early as possible, but they were still difficult to identify, because manually examining parsing states was very tedious. So finally, the last step was to write a result tester by hand that examined the parser states to give hints. And because the library is written as plain OCaml functions and combinators, it was very easy to tweak just the right part.

Modules. The last week of the MOOC was dedicated to the module language. Learners learned about wrapping types and values in modules, giving them interfaces, and using the parametric modules of the standard library.

To grade exercises that require the learner to define modules, we simply use the first class module mechanism of OCaml. When the get_value introspection primitive is called with an uppercase identifier and a first class module type witness, instead of looking for a variable, it looks for a module. If it is found, it performs a structural inclusion test and boxes it in a first class module.

Figure 12 shows an example grader. First, it defines the expected signature. Then it tries to access the learner's module under that type. If either the lookup or the type check fails, an error is reported. Otherwise, the grader continues, using the same techniques as for normal toplevel functions.

This week was also the occasion to teach abstract data types. Some exercises had to grade functions that worked on types hidden by the learner, and thus unknown to the grader. In cases like these, the automatic random samplers and printers are useless. Indeed, most of our graders work by comparing the results of the learner's functions to our solutions', which cannot be done if the two parties work on values of their own types.

To preserve this approach of comparing the behaviour of the learner's code to the solution, we had to bend the grading paradigm a little bit. The solution we used was to generate code that internally works on abstract values but whose inputs and outputs are of concrete types, and to run this code over both implementations. As a trivial example, suppose a module with an abstract type $t$ and two functions to_string: $t \rightarrow$ string and from_string: string $\rightarrow t$. Testing these functions individually is not possible since they use values of type $t$, which may be implemented differently by the learner and in the solution. So a workaround is simply to check (fun $s \rightarrow>$ to_string (from_string s)). As a more realistic example, one of the exercises of the MOOC was to implement an associative table. What we did was generating code that would create an empty table, perform a random sequence of insertions and deletions, and then a final lookup.

In practice, to generate this testing code, rather than forging an AST, we simply used plain OCaml functions. We started by defining the basic operations as functions that take as input the module to test. Then, we sequence them using combinators, to obtain the test procedure as a plain 
OCaml function that takes the module to test as argument. A big advantage is that the generated test code is as well typed as the rest of the grader.

Sensible random sampling. As motivated in Sections 3 and 4, we wanted the automatic grader and its reports to be central in the workflow of learners. They would just run the grader and correct their code in a cycle until their reports became completely green. This put lots of constraints on the test cases.

First, they had to be exhaustive, to actually find bugs. They also had to be pertinent, to pinpoint as precisely as possible the locations of errors. Then, they had to cover properly the possible outcomes so that, for instance, a boolean function would have been given test cases ending up in as many true results than false ones. Along with these choices, we also had some external constraints. The first one was basic cheating prevention, which meant that hardcoding test cases was not an option, so we had to generate them. And since everything runs inside the browser, it meant that generation had to be fast. To satisfy these constraints, the decision was made to sample around ten random test cases per question. Thus, in a lot of cases, the default automatic random samplers are not satisfactory. And with as few as ten test cases, we felt we had no real other choice than resorting to manual random sampler writing, with the aid of combinators. For instance, one of the exercises of the MOOC asked the learners to write a function balanced over binary trees. What we did was writing a sampler for balanced trees, and another for unbalanced ones. After this manual part, the rest was handled by the sample_alternatively combinator, which produces a distribution with an equal amount of both, in an order that is not predictable by the learner.

In the end, this choice was very conclusive in terms of results and learner satisfaction, but probably at the cost of too much effort on our side. We plan to look for a more principled and if possible less manual approach for the next versions of the grading library.

Type definitions. When writing programming exercises for beginners, it is usual to decompose a complex problem into smaller questions; and in a functional programming course a typical question is to ask learners to define a new data type. Such a question is not easy to grade automatically.

If we want grade a type definition per se, one might try to syntactically compare the proposed type definition with some expected definitions. But this is quite restrictive and we might reject some acceptable type definitions we did not think of when writing the exercises. Even if we try to add more flexibility in the grader by using some clever type-isomorphism-detection algorithm, this is still a zero-or-full-grade situation, and this might be quite frustrating for creative learners.

What we have done during this MOOC is usually to provide the type definition in the prelude and only to grade the functions handling this data type. However, in some more advanced problems, we let the learners write their own data type, yet we did not try to grade the definition. We rather considered it as an abstract type, then we graded the problem as a whole, in "black box" mode. For instance, in a problem where the students had to write their own data structure for sets of integers, the grader handled the add function by inspecting its results using the cardinal or mem functions.

(Human) feedback. A remark, appearing multiple times in the MOOC's forum, is the lack of feedback once a learner reaches the maximum score of a given exercise. While happy to finally handle all the test cases, the learners were willing to know, for instance, whether there solution was "idiomatic", "concise enough", or "reasonably efficient compared to the others learners"”. And indeed, we believe there is a pedagogical interest in reading someone-else's code after having spent a lot of time in writing one's own solution. If some discussions between learners in the forum partially fulfilled these requests, we also wish to improve the grader to not only list failing-and-passing unit tests, but also give some stylistic advice or some bonus points when a given pattern is used. And, 
for exercises where there exists some idiomatic solution, we might also decide to include it in a successful report.

Another improvement that we might do in grading report is to annotate hand-written tests with explanations on why we believe this test is pertinent and which classical errors might let this test fail. These kinds of hints might help learners to not lose interest in a difficult exercise where they might feel a bit lost. However, writing all these annotations is time consuming, and we are not sure it is always worth the added value: there is also an interest in letting learners deconstruct a failing test and understand their mistake. We believe such hints should not be mandatory; it is just a matter of finding the right balance and we have yet to review each problem individually in order to improve the ones that were reported as far too difficult or time-consuming with better hints.

\section{WRITING THE OCAML MOOC IN OCAML}

The platform on which the OCaml MOOC was hosted relies on the Open edX software stack. As most MOOC software, it features a course player for learners, and a WYSIWYG course editor for teachers. These components communicate via a common course archive format, that is also used to download and restore backups.

Quite soon in the development of the MOOC, we understood that the WYSIWYG editor was nothing more than an obstacle. Indeed, as computer scientists, the nice level of user friendliness and abstraction provided was not necessary for us, whereas the important amount of clicks necessary to perform basic tasks very frustrating. A lot of operations felt really repetitive, or in other words, could have been automated. So we decided to produce the internal archives offline and use the backup system to upload them. And of course, instead of forging these archives by hand, we decided to build them using an OCaml program. It takes the form of an embedded DSL to describe courses, and a function that compiles a description to a backup archive.

Lots of static checks are performed, so that most mistakes are caught as soon as possible. Most of the checks are just to produce a well formed archive. The grammar of all XML and JSON files is checked. All the IDs in these files are generated in a well-thought way, so that they do not collide or change during an update (which would lead to data loss or mixed up URLs). The references to bundled files, other course pages, external URLs and IDs are checked, so that no broken link is created. Some semantics of meta information is also verified, such as the sequence of dates being monotone, or the sum of all grades adding up to the expected $100 \%$. We also wrote checks that are specific to this MOOC. For instance, each exercise grader is compiled and checked before inclusion, and its maximum score is computed using our reference solution.

In the end, the amount of static guarantees lowered the required testing to almost zero, and allowed us to update the MOOC in place in production without hesitation, even for fixing small typos, without even thinking about it. It may not seem that impressive, but to put things into perspective, all other teachers we know who used the same platform told us that they were really reluctant to touch anything once their course was online. Most of the checks listed above are not done at all when updating via the editor or importing an archive, potentially leading to broken or wrongly formatted course contents, and in not-so-extreme cases, data loss, including grades.

This level of automation also gave us the ability to implement features that would have been too time consuming or simply impossible if we had used the standard tools. For instance, our compiler automatically generates per-week course archives for learners, and a searchable table of contents. 
Finally, the level of abstraction of our DSL unties us almost completely from the platform's format, so we will be able to deploy the OCaml MOOC somewhere else in the future, if that becomes necessary. So for all these reasons, we clearly recommend anyone working on a programming MOOC to give this approach a try.

The amount of code necessary to implement the DSL was reasonable compared to the actual description of the MOOC, as shown in Figure 23 at the end of the article, yet it was not negligible, so we would be happy if it could serve others' needs.

\section{STATISTICS}

The two first sessions of the OCaml MOOC started respectively in October 2015 and September 2016. Both lasted for seven weeks, numbered from 0 to 6 , and the final date for the submission of the project was set at the end of each year. A final note was computed by assigning each week from 1 to 6 a maximum score of 0.1 points, and a maximum of 0.4 points to the project. To obtain the certificate, and pass the course, a note greater or equal to 0.6 was required.

The amount of work required from the students was high. There were 48 exercises for the main six weeks of the course, ranging from warm ups to advanced higher order functional manipulations of algebraic data structures, including trees and lists. For the final project, a choice was offered between two subjects: writing a solver for the popular Klotski puzzle, or building Markov chains from a text corpus for generating amusing text fragments. Each of them required writing a couple of hundred well thought lines of OCaml code.

At the end of the course, we asked the students to fill a detailed questionnaire to collect their feedback on all parts of the course, including the final project. In the first year, we collected 288 answers from both students that passed, and students that did not pass the course, representing over $20 \%$ of the active population. Unfortunately, we could not process the second year's questionnaire due to new privacy restrictions imposed by the FUN platform in 2016, that prevented us from linking the questionnaire's answers to the grades.

Participation. There have been over 7000 learners enrolled (more than 3500 each year) from more than 120 countries (France, US, Spain, UK, India and Germany being the top 6, the global distribution is represented in Figure 13). Of these, 2418 actually showed up when the course started (those we consider active in the rest of this section).

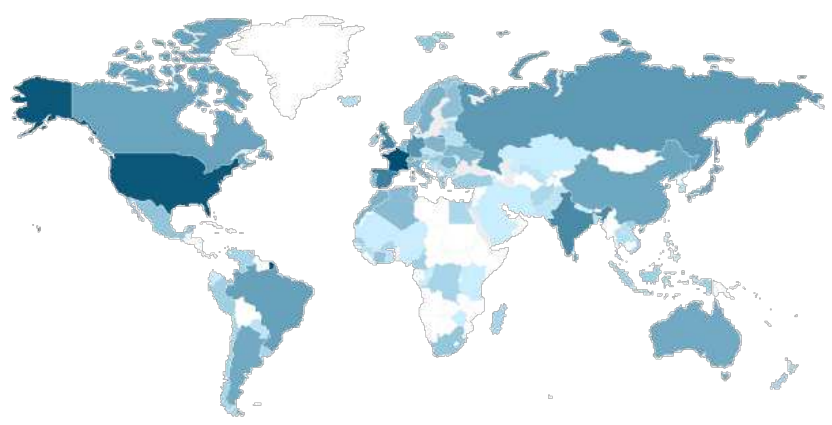

Fig. 13. Map of participation by country 
The evolution of the participation, from the enrollment phase to the final project, is shown in Figure 14: remarkably, the learners that stayed more than two weeks essentially kept up with all the course. At the end, 588 obtained a certificate for the course (318 the first year and 270 the second year), which is $8.42 \%$ of the enrolled population, and $24.32 \%$ of the active population.

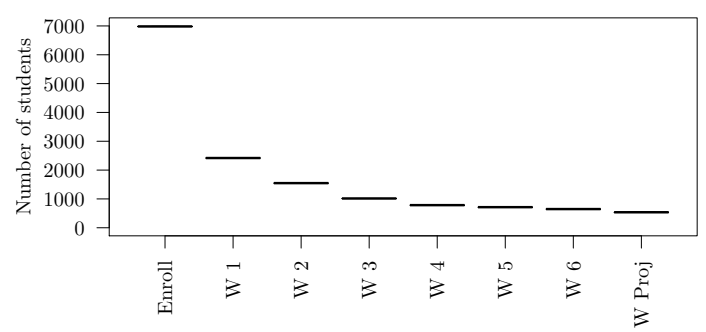

Fig. 14. Evolution of participation

In the following, we perform a statistical analysis over the data available for the 2418 active students (1250 the first year, 1168 the second year), and the 288 respondents to the questionnaire of the first year.

Demographics. As shown in Figure 15, the 2418 activestudents span a very wide spectrum of ages, with the highest density in the 20 to 35 age range, and a long tail reaching up to 80 years old.

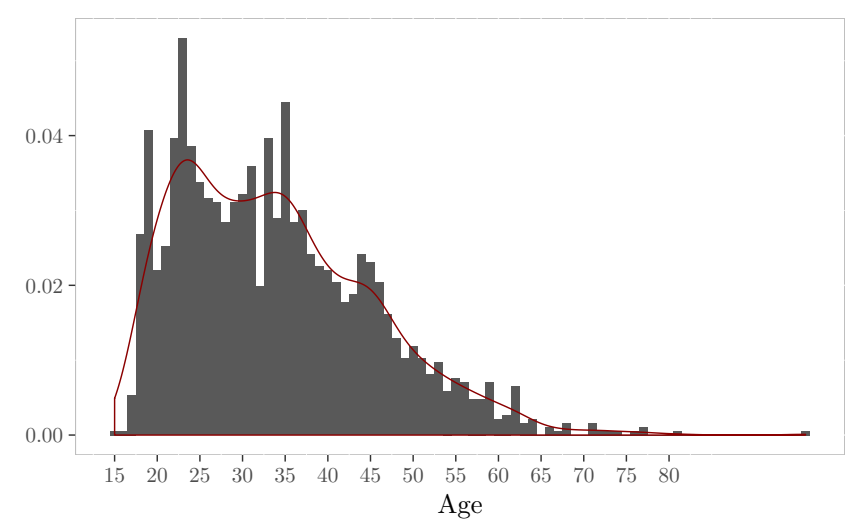

Fig. 15. Distribution of ages of active learners

On the gender side, there is much less diversity, with only 142 women having actively participated.

An interesting question concerns the relevance of the degree of the participants on their engagement and results. The information in Table 16, together with the age distribution, shows that there is a significant portion of participants that are still students, which is unusual for this kind of MOOCs, where traditionally one finds a large majority of working professionals. Unsurprisingly, the highest success rates are also concentrated at the undergraduate and graduate level. 


\begin{tabular}{|c|c|c|c|c|}
\hline & \multicolumn{2}{|c|}{ Active } & \multicolumn{2}{|c|}{ Passed } \\
\hline & Numbers & avg. success & Numbers & avg. success \\
\hline High School & $\square 255$ & 20.93 & I 32 & 90.19 \\
\hline Associate & I 45 & 21.29 & 14 & 86.50 \\
\hline Bachelor & 567 & 口 $\quad 26.70$ & 113 & 87.12 \\
\hline Master & 775 & $\begin{array}{l}\mathbf{0} \quad 37.13\end{array}$ & 242 & 86.69 \\
\hline $\mathrm{PhD}$ & $\square 226$ & $\begin{array}{l}\mathbf{\square} \\
\mathbf{0}\end{array}$ & 口 75 & 91.44 \\
\hline Elementary & 17 & 口 26.43 & $\mid 1$ & 100.0 \\
\hline Junior High & I 65 & 口 $\quad 21.14$ & 19 & 85.44 \\
\hline Other & I 40 & $\begin{array}{ll}\mathbf{m} & 34.02\end{array}$ & I11 & 86.45 \\
\hline $\mathrm{N} / \mathrm{A}$ & 438 & ש $\quad 34.93$ & 128 & 89.41 \\
\hline
\end{tabular}

Fig. 16. Degree distribution and rate of success

The data points at the Elementary and Junior High level seem to be data entry mistakes, as most of these learners declare ages over 20, and we drop them in what follows. Similarly, we drop the unfortunately significant pool of people who chose to keep their level of education to themselves, or did not find their diploma in the list.

Distribution of scores. Focusing now on the 648 learners that were active until the last week of the course, we can see in Figure 17 that almost all of them passed (the pass note was at 0.6), and, independently from the degree, the distribution is very skewed towards the maximum, with a median which is extremely high. This confirms the appropriateness of our choice of leaving open the submission of the exercises until the end of the course, and with no limit on the number of trials: as can also be seen looking at the messages on the @ocamlmooc Twitter feed, the learners that sticked to the course made a point of honour to try and get $100 \%$ of all the homework right.

This is precisely the goal we had fixed for the OCaml MOOC: we did not want to rank students, but bring as many newcomers as possible to a high proficiency level in functional programming. As is clearly shown in Figure 18, about 290 learners got a perfect score, achieving results comparable to the very best students in the courses we run the traditional way.

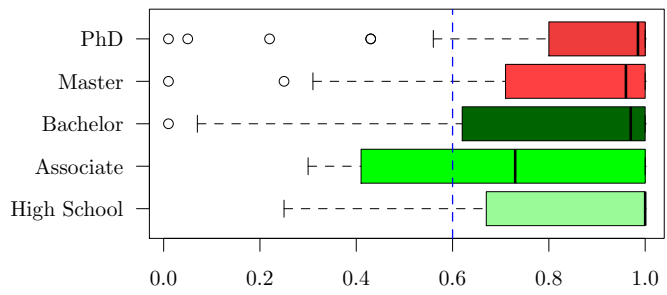

Fig. 17. Distribution of scores per degree of students that stayed to the last course

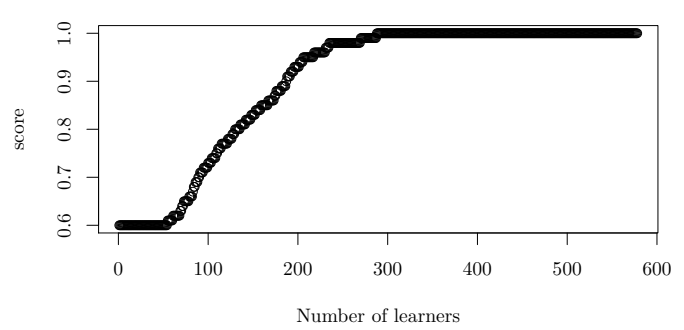

Fig. 18. Scores of students that stayed to the last course

Perceived effort and course appreciation. The FUN platform offered no means to track the effort spent by the learners on the course, nor the perceived difficulty level or their overall appreciation. Hence we resorted to a questionnaire. The first year, there were 288 respondents, 118 of which did not get a certificate, and 169 which got the certificate; 163 of them found the difficulty just right, 88 found it difficult, 23 easy, 3 too easy and 10 too difficult. 
In Figure 19 we see that the perceived difficulty is clearly correlated to the median value of the final result (except the marginal case of the too easy), but the individual performance varies widely, and even learners that found the course easy or just right did not necessarily perform well.

In Figure 20, the time spent per week on the course appears perfectly correlated in the respondents' questionnaire with the perceived difficulty of the course.

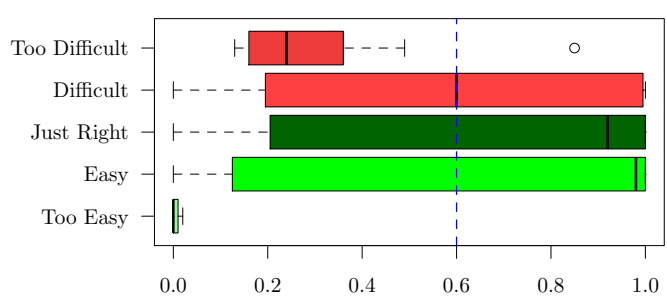

Fig. 19. Distribution of scores (0 to 1$)$ per perceived difficulty

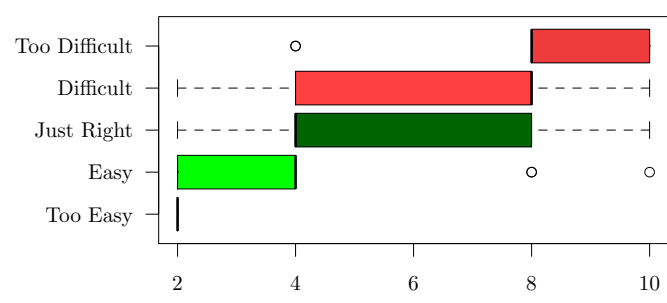

Fig. 20. Weekly effort (hs/week) per perceived difficulty

The median amount of work expected for a student that finds the course just right is 4 hours per week, and not the 2 hours per week that were advertised in the first year: this information has been used in the second year to update the course description.

Another interesting question to explore is how the overall appreciation of the course quality is related to the degree (Figure 22) and the final score (Figure 21).

Unsurprisingly, we see in Figure 21 that the appreciation of the course and the grades obtained by the learners are correlated.

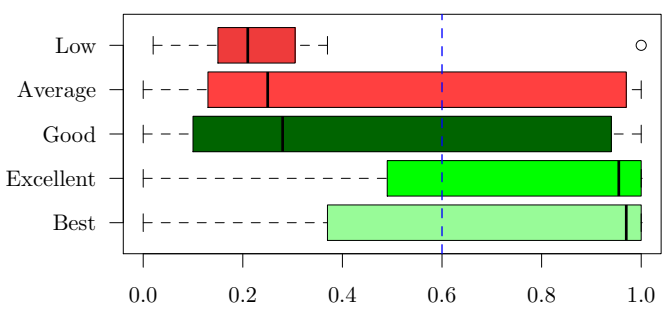

Fig. 21. Course appreciation vs. final score

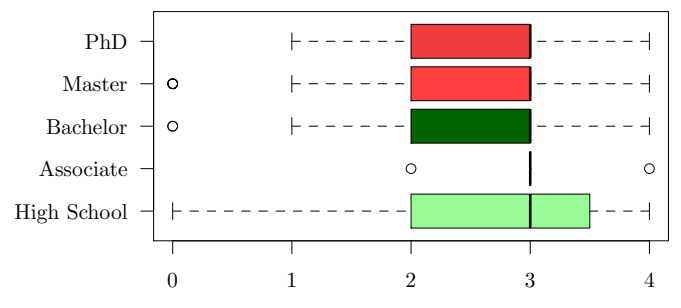

Fig. 22. Distribution of appreciation per learner degree

On the other hand, we were quite satisfied to clearly see in Figure 22 that the distribution of the appreciation of the course is quite the same for the learners with at least an introductory undergraduate degree, which was the expected background for the OCaml MOOC enrolled learners.

\section{RELATED WORK}

There has been extensive work on teaching programming using different programming languages, tooling and approaches, and it would be impossible to survey here all the related literature, so we focus here on related work that is relevant to teaching functional programming, in particular in a massive online course.

The most famous and successful MOOC introducing functional programming is without doubt the one run in Scala by Martin Odersky [Miller et al. 2014], that over the years has attracted over 
100.000 learners. The approach taken in that course, though, is radically different from ours, as it requires the learners to install the full environment before starting the course, and it relies on a full server-side infrastructure to evaluate the student code. The same holds for the MOOC on functional programming by Erik Meijer on Haskell ${ }^{5}$.

Many projects have recognised the value of giving the learners a "zero-install" environment for a seamless programming experience : we can mention WeScheme [Yoo et al. 2011] and PyRet, dedicated to functional programming in Scheme, the Code.World environment developed in Haskell, and the MOOCs Introduction to interactive programming in Python ${ }^{6}$ from Rice University and Python: des fondamentaux à l'utilisation du langage ${ }^{7}$ from Inria dedicated to Python; there are also numerous initiatives that try to attract young students to programming, like [Papancea et al. 2013; Piccioni et al. 2014] and notably the FranceIoI ${ }^{8}$ portal, that presents exercises that can be solved in C, C++, Pascal, OCaml, Java, JavaScool and Python, similarly to the widely used PythonTutor.

All these approaches give the learners the illusion to run their code directly in their browser, but in reality are all built on the same traditional client-server architecture, with a web front-end that make calls behind the scenes to an interpreter or compiler, with the related risks of potential downtime, server overload, or server compromission.

Our approach is totally server-less, and what is shown to the learners is not an illusion, but the reality: their code is compiled and run precisely inside their browser. This has been made possible by the availability of the OCaml to JavaScript compiler [Vouillon and Balat 2014], but all the other developments are new.

Automatic grading is also a thoroughly explored research area. Since the seminal article by Hollingsworth [Hollingsworth 1960], a wealth of approaches have been experimented and refined to make programming training scalable. Some more recent work can be found in [Singh et al. 2013] and [Jeuring et al. 2014].

The originality of our work comes from a combination of techniques: on one side, we generate automatically test cases for complex data structures based on their types, following the tradition started by QuickCheck [Claessen and Hughes 2000]; on the other, we build on this a type-safe environment for testing the learners code directly inside their own browser.

To the best of our knowledge, when taking into account all of its facets, the complete environment provided by this MOOC is unique, and has been one of the main reasons of the high degree of satisfaction of the thousands of learners that have followed this course.

\section{CONCLUSION}

The "Introduction to functional programming in OCaml" MOOC was specifically designed and implemented with the goal to retain learners all along the course, and bring them to a high level of proficiency, in functional programming and in the OCaml programming language.

To this end, the course content has been carefully designed to provide a step by step introduction to the basic notions of functional programming, delaying on purpose to the last courses the presentation of more language specific traits, like imperative traits (input/ouput, exceptions and mutable data) and modules and compilation units.

To support this approach, a full fledged development environment has been developed that allows learners to write, check and execute medium sized programs directly in a web browser, avoiding all installation hassles, and going well beyond the Ajax-based web consoles interfacing with remote execution servers that are commonplace nowadays.

\footnotetext{
${ }^{5}$ https://www.edx.org/course/introduction-functional-programming-delftx-fp101x-0

${ }^{6}$ https://www.class-central.com/mooc/408/coursera-an-introduction-to-interactive-programming-in-python-part-1

${ }^{7}$ https://www.fun-mooc.fr/courses/inria/41001S03/session03/about

${ }^{8}$ FranceIoI : Le site d'entraînement à la programmation et l'algorithmique http://www.france-ioi.org/index.php
} 
Exercise platform:

\begin{tabular}{|l|c|l|}
\hline Component & Lines & Type \\
\hline \hline Report library & 500 &. $\mathrm{ml}$ \\
\hline Grading library & 1500 &. $\mathrm{ml}$ \\
\hline Exercises and projects & 4700 &. $\mathrm{ml}$ (tests) \\
& 1500 &. $\mathrm{ml}$ (solutions) \\
& 800 &. $\mathrm{ml}$ (templates) \\
& 4000 &. $\mathrm{html}$ (descriptions) \\
\hline Offline exercise and grader checker & 500 & $. \mathrm{ml}+$ Makefile \\
\hline Adapted Try OCaml & 2000 &. $\mathrm{ml}$ \\
\hline User Interface & 1500 &. $\mathrm{ml}$ \\
\hline
\end{tabular}

MOOC description and deployment DSL:

\begin{tabular}{|l|c|l|}
\hline Component & Lines & Type \\
\hline \hline Generic DSL and deployment library & 2000 & $. \mathrm{ml}+. \mathrm{mli}$ \\
\hline OCaml MOOC specific layer & 1000 &. $\mathrm{ml}$ \\
\hline OCaml MOOC description & 1500 &. $\mathrm{ml}$ \\
\hline
\end{tabular}

Fig. 23. Volume of code for the complete MOOC

Testing and grading the user programs locally inside the learner's browser is to the best of our knowledge entirely new, as is the library that allows easy and safe writing of test cases.

The combination of these three innovations has allowed the learners that sticked to the course to obtain an outstanding level of proficiency, with 588 out of the 2418 active learners going through all the exercises and the final programming projects, and about 300 getting $100 \%$ of the final score. Obtaining the same results using the traditional teaching approach followed in a regular course with comparable content in local Universities would require, in our experience, repeating the course for the equivalent of more than twenty years.

Hence, we consider this experience a definite success, and we hope the lessons learned and reported here will be helpful to other MOOC designers interested in bringing quickly a large amount of learners to a high degree of proficiency in programming.

\section{SOURCE CODE AVAILABILITY}

Figure 23 shows the number of OCaml lines of code written for supporting the full OCaml MOOC.

The source code of the whole exercices platform developed for the MOOC, containing the enhanced code editor based on ACE, the toplevel, and the automatic grader has been released as Open Source and a standalone demo in which a few exercises can be tried is available at https://try.ocamlpro.com/fun-demo/.

\section{ACKNOWLEDGEMENTS}

The OCaml MOOC would not have been so successful without the collaborative effort of many people: Ralf Treinen and Yann-Regis Gianas spent countless hours working with the second author on the content and the videos of the course; Çagdas Bozman contributed significantly to the special version of TryOCaml used in the exercise environment; a team of volunteers at Irill and LIP6, with special thanks to Emmanuel Chailloux, produced the english subtitles, and Jean-Francois Monin their french translation. Special thanks go to Avner Bar-Hen, that has already run twice a MOOC on statistics: his guidance and advice have been precious in the statistical analysis of the data collected during the OCaml MOOC. 


\section{REFERENCES}

Erik Barendsen and Valentina Dagiene (Eds.). 2014. Proceedings of the Computer Science Education Research Conference, CSERC 2014, Berlin, Germany, November 5-6, 2014. ACM. https://doi.org/10.1145/2691352

Hans-Juergen Boehm and Cormac Flanagan (Eds.). 2013. ACM SIGPLAN Conference on Programming Language Design and Implementation, PLDI '13, Seattle, WA, USA, June 16-19, 2013. ACM. http://dl.acm.org/citation.cfm?id=2491956

Randal E. Bryant, Klaus Sutner, and Mark J. Stehlik. 2010. Introductory Computer Science Education at Carnegie Mellon University: a Deans' Perspective. Technical Report CMU-CS-10-140. School of Computer Science, Carnegie Mellon University.

Koen Claessen and John Hughes. 2000. QuickCheck: a lightweight tool for random testing of Haskell programs, See [Odersky and Wadler 2000], 268-279. https://doi.org/10.1145/351240.351266

Robert Dewar and Owen L. Astrachan. 2009. Point/counterpoint - CS education in the U.S.: heading in the wrong direction? Commun. ACM 52, 7 (2009), 41-45.

Roberto Di Cosmo, Yann Regis-Gianas, and Ralf Treinen. 2015. Introduction to functional programming using the OCaml programming language. https://www.fun-mooc.fr/courses/parisdiderot/56002/session01/about. (October 2015).

Matthias Felleisen, Robert Bruce Findler, Matthew Flatt, Shriram Krishnamurthi, Eli Barzilay, Jay A. McCarthy, and Sam Tobin-Hochstadt. 2015. The Racket Manifesto. In 1st Summit on Advances in Programming Languages, SNAPL.

Vincent Gramoli, Michael A. Charleston, Bryn Jeffries, Irena Koprinska, Martin McGrane, Alex Radu, Anastasios Viglas, and Kalina Yacef. 2016. Mining autograding data in computer science education. In Proceedings of the Australasian Computer Science Week Multiconference.

Grégoire Henry, Michel Mauny, Emmanuel Chailloux, and Pascal Manoury. 2012. Typing Unmarshalling Without Marshalling Types. SIGPLAN Not. 47, 9 (Sept. 2012), 287-298.

Jack Hollingsworth. 1960. Automatic Graders for Programming Classes. Commun. ACM 3, 10 (Oct. 1960), 528-529. https://doi.org/10.1145/367415.367422

Johan Jeuring, L. Thomas van Binsbergen, Alex Gerdes, and Bastiaan Heeren. 2014. Model solutions and properties for diagnosing student programs in Ask-Elle, See [Barendsen and Dagiene 2014], 31-40. https://doi.org/10.1145/2691352. 2691355

Ville Karavirta, Ari Korhonen, and Lauri Malmi. 2006. On the use of resubmissions in automatic assessment systems. Computer Science Education 16, 3 (2006), 229-240.

Mikko-Jussi Laakso, Tapio Salakoski, Linda Grandell, Xuemei Qiu, Ari Korhonen, and Lauri Malmi. 2005. Multi-Perspective Study of Novice Learners Adopting the Visual Algorithm Simulation Exercise System TRAKLA2. Informatics in Education 4, 1 (2005), 49-68.

Heather Miller, Philipp Haller, Lukas Rytz, and Martin Odersky. 2014. Functional programming for all! scaling a MOOC for students and professionals alike. In 36th International Conference on Software Engineering, ICSE.

Robin Milner. 1978. A Theory of Type Polymorphism in Programming. f. Comput. Syst. Sci. 17, 3 (1978), 348-375.

Dejan S. Milojicic. 2011. Autograding in the Cloud: Interview with David O’Hallaron. IEEE Internet Computing 15, 1 (2011), 9-12.

Martin Odersky and Philip Wadler (Eds.). 2000. Proceedings of the Fifth ACM SIGPLAN International Conference on Functional Programming (ICFP '00), Montreal, Canada, September 18-21, 2000. ACM.

Andrei Papancea, Jaime Spacco, and David Hovemeyer. 2013. An open platform for managing short programming exercises. In International Computing Education Research Conference, ICER.

Marco Piccioni, Christian Estler, and Bertrand Meyer. 2014. SPOC-supported Introduction to Programming. In Proceedings of the 2014 Conference on Innovation \&\#38; Technology in Computer Science Education (ITiCSE '14). ACM, New York, NY, USA, 3-8. https://doi.org/10.1145/2591708.2591759

David Pritchard and Troy Vasiga. 2013. CS circles: an in-browser python course for beginners. In Proceeding of the 44th ACM technical symposium on Computer science education, SIGSCE.

Christian Queinnec. 2010. Invited Talk : On Automated Grading. In fournées francophones des langages applicatifs (FFLA). http://www.paracamplus.com/.

Guido Rößling, Thomas L. Naps, and Christian Spannagel (Eds.). 2011. Proceedings of the 16th Annual SIGCSE Conference on Innovation and Technology in Computer Science Education, ITiCSE 2011, Darmstadt, Germany, fune 27-29, 2011. ACM.

Rishabh Singh, Sumit Gulwani, and Armando Solar-Lezama. 2013. Automated feedback generation for introductory programming assignments, See [Boehm and Flanagan 2013], 15-26. https://doi.org/10.1145/2462156.2462195

Jérôme Vouillon and Vincent Balat. 2014. From bytecode to JavaScript: the Js_of_ocaml compiler. Software: Practice and Experience 44, 8 (2014), 951-972.

Danny Yoo, Emmanuel Schanzer, Shriram Krishnamurthi, and Kathi Fisler. 2011. WeScheme: the browser is your programming environment, See [Rößling et al. 2011], 163-167. https://doi.org/10.1145/1999747.1999795 